\title{
ARTICLE
}

\section{Clinical staging models: from general medicine to mental disorders}

\author{
Jan Scott \& Chantal Henry
}

Jan Scott is Professor of
Psychological Medicine at

Newcastle University, a Visiting

Professor at the Institute of

Psychiatry, Psychology and

Neuroscience in London, the

University of Sydney, and the

Norwegian University of Science

and Technology in Trondheim, and

a Science Without Borders Fellow

in Brazil (Sao Paulo and Porto

Alegre). She is also a Distinguished

Founding Fellow of the Academy

of Cognitive Therapy. Her recent

research includes prospective

studies of risk of bipolar disorders

and clinical staging models; new

methods of analysis of sleep-wake

cycles in youth with emerging

mood disorders; and the design and

piloting of preventive interventions

for young people at risk of bipolar

disorders. Chantal Henry is

Professor of Psychiatry at the

University of Paris-Est and has a

position in the Pasteur Institute,

Paris. She coordinates the French

network of expert centres for bipolar

disorders. Her research is focused

on a dimensional approach to

better characterise mood episodes

and find relevant biomarkers

from a translational perspective

(from rodents to humans).

Correspondence Professor Jan

Scott, Academic Psychiatry, Wolfson

Unit, Campus for Vitality \& Ageing,

Westgate Road, Newcastle upon

Tyne NE4 6BE, UK. Email: jan.scott@

newcastle.ac.uk

\section{Copyright and usage}

(C) The Royal College of Psychiatrists 2017.

\begin{abstract}
SUMMARY
Revisions of international classification systems for mental disorders have focused on improving the reliability of diagnostic criteria. However, the uncertain validity of the current diagnostic categories means that they do not always fulfil their key purposes, namely to guide treatment and predict outcomes. This is especially true when traditional diagnostic approaches are applied to adolescents and young adults with emerging illnesses. A clinical staging model, similar to those used in general medicine, could improve diagnosis in psychiatry and aid treatment decision-making, especially if applied to individuals aged about 15-25 years, which is the peak age range for the onset of severe mental disorders. Staging models may offer a new framework for the development of interventions with high benefit and low risk, and for research into neurobiological and psychosocial risk factors. However, this approach is not without controversy: some experts oppose its introduction, some argue that it represents a transdiagnostic model, and some suggest it is only viable if disorder-specific models are used.
\end{abstract}

\section{LEARNING OBJECTIVES}

- Gain awareness of some limitations of current approaches to psychiatric diagnosis

- Review the basic principles of clinical staging models used in general medicine

- Understand current research on the use of staging models in psychiatry, and attempts to apply these models to bipolar disorders

\section{DECLARATION OF INTEREST}

None

The word 'diagnosis' originates from the Greek words dia (apart) and gignokein (to recognise or know). In any medical specialty, the first step in making a diagnosis is a clinical assessment interview (including the history of the presenting complaint, course of symptoms and illness, a detailed review of risk and protective factors, and family history). In branches of medicine other than psychiatry, a range of investigations can be used to aid diagnosis. The absence of laboratory tests in psychiatry means that the diagnosis of a mental disorder relies on clinical judgement and recognition of patterns of symptoms observed or reported in the mental state examination. There are two main problems with this. First, applying a psychiatric diagnosis often represents an attempt to impose a 'present/absent' or 'yes/ no' classification on a clinical presentation that, in reality, is dimensional and varies over time. Historically, this problem was compounded by a lack of international consistency in diagnostic categories for mental disorders, and it was often unclear whether different groups were communicating about and comparing the same clinical conditions. Second, although diagnosis is essential to high-quality treatment in every branch of medicine, there is a lack of consensus on the thresholds for psychiatric treatment or for deciding which treatment to use for any given diagnosis.

Over the past three decades, the development of internationally agreed, operationally defined criteria for mental disorders - such as the DSM and ICD - has facilitated a significant improvement in the reliability of diagnosis in psychiatry. However, a major problem with the current diagnostic categories for severe mental disorders is that they do not have adequate predictive validity (McGorry 2002; Insel 2009; Hickie 2013; Scott 2013). This is partly owing to the methods used for developing the diagnostic criteria. For example, the concepts described in the recent revisions of both the DSM and the ICD for the three most prominent severe mental disorders in adults (major depression, psychosis and bipolar disorders) were mainly developed from cross-sectional observations of clinical samples of middle-aged adults with longestablished, chronic syndromes that were being treated in mental health settings (Scott 2013). Several problems can arise when these diagnostic criteria are applied to young people with less stable, less well-defined syndromes (McGorry 2006). Also, at an earlier stage in the illness, the clinical features of one disorder may overlap substantially with those of other severe disorders, or the most prominent presenting symptoms may not be those that most clearly define the established phase of the disorder (McGorry 2002, 2006). For example, Duffy (2010) noted that several longitudinal 
prospective studies of the offspring of parents with bipolar disorder demonstrate clearly that these children rarely present to clinical services for the first time with full syndromal bipolar disorder. The more likely picture is that of heterotypic continuity, namely that the offspring often report a range of non-specific antecedents in childhood (e.g. anxiety and sleep problems), which may progress to more specific mood disturbances in late adolescence or early adulthood, with depressive episodes usually preceding a first manic episode (Duffy 2010; Loftus 2016).

Current approaches to diagnosis fail to take into account the trajectory of the illness. Consequently, a young person can potentially accumulate several diagnoses over time as an adult-pattern mental disorder gradually evolves. A traditional approach to diagnosis would give the impression that the person has had multiple lifetime comorbid disorders, creating a phenomenon that is often referred to as artefactual comorbidity (Loftus 2016). In reality, in most cases this clinical picture describes an individual who is predisposed to develop a severe mental disorder such as bipolar disorder in early middle adulthood; in the early course of this the manifestation of a range of symptoms of anxiety disorder not otherwise specified (NOS), attention-deficit hyperactivity disorder (ADHD) NOS, adjustment disorder, depression NOS with cyclothymia and/or bipolar disorder is typical. This pattern of heterotypic continuity from childhood to adolescence and then to early adulthood is also seen in birth cohorts, and several prospective community studies have demonstrated that psychosis, major depression and bipolar disorders often have similar patterns of disorder or illness trajectories prior to the onset of a syndrome that meets the traditional diagnostic criteria of a severe mental disorder (Kim-Cohen 2003).

Owing to the frequency with which the above problems are encountered with the current diagnostic framework, several research groups have examined alternative approaches to classifying and managing mental disorders, especially the potential application of clinical staging models (McGorry 2006; Berk 2007; Hickie 2013; Scott 2013; Treasure 2015; Verduijn 2015).

\section{Staging models in general medicine}

In physical disorders such as cancer and diabetes, clinical staging models are routinely used to enhance the early detection and systematic management of the disease process (Gonella 1984). Some of the key elements of staging are shown in Box 1. The earliest references to the concept of
BOX 1 Basic assumptions of clinical staging models

According to Gonella et al (1984), staging defines 'discrete points in the course of a disease' that:

- are clinically detectable

- reflect severity in terms of risk of residual impairment or death

- are clinically significant predictors of illness prognosis or the most appropriate therapeutic modality.

clinical staging models can be found in publications on the diagnosis and treatment of cancer. For example, in the 1920s, the work of Albert Broders on the numerical grading of tumours played an important part in the widespread adoption of staging in medicine (Wright 2012). However, clinical staging was soon recognised as having utility in a wide range of chronic conditions, including ischaemic heart disease (IHD), Parkinson's disease and rheumatoid arthritis. Critically, staging models incorporate the notion of disease extension (evolution in terms of both the range of symptoms and the bodily systems involved) and describe where an individual exists on a continuum from an asymptomatic, at-risk state through to early illness, and onwards to end-stage disease (McGorry 2006; Scott 2013). Staging actively facilitates predictions about disease progression that are largely independent of illness severity or duration. Overall, it is a more refined concept than cross-sectional diagnosis, as the latter is largely blind to concepts such as age at onset or illness trajectories.

Table 1 shows the key clinical stages of IHD. Individuals at high risk of IHD (i.e. those at stage 0 , also called the at-risk or latency stage) can be identified by the presence of known risk factors found during routine screening, such as high nicotine consumption, raised plasma lipid

TABLE 1 Clinical staging model of ischaemic heart disease: an example of illness and treatment progression

\begin{tabular}{|c|c|c|}
\hline Stage & Common characteristics & Typical interventions \\
\hline $\begin{array}{l}0 \text { (at-risk or } \\
\text { latency stage) }\end{array}$ & $\begin{array}{l}\text { Increased risk: family history (clinical } \\
\text { examination, genetic tests) }\end{array}$ & $\begin{array}{l}\text { Try to minimise risk, encourage } \\
\text { healthy diet and exercise }\end{array}$ \\
\hline 1 & Obesity, smoking, high cholesterol & Use diet and exercise plus statins \\
\hline 2 & Increased blood pressure & $\begin{array}{l}\text { Use anti-hypertensives, e.g. beta- } \\
\text { blockers }\end{array}$ \\
\hline 3 & Angina & $\begin{array}{l}\text { Use anti-angina drugs, e.g. glyceryl } \\
\text { trinitrate }\end{array}$ \\
\hline $\begin{array}{l}4 \text { (late or } \\
\text { end stage) }\end{array}$ & Heart attack (myocardial infarction) & $\begin{array}{l}\text { Surgery to insert cardiac stents } \\
\text { (to bypass damaged arteries) }\end{array}$ \\
\hline
\end{tabular}


levels or family history of IHD. It is noteworthy that, even at this pre-symptomatic stage, lifestyle interventions such as exercise and diet changes are often recommended to try to reduce the risk of progression to IHD. In some individuals, these approaches may be combined with specific medical therapies such as the selective use of statins to reduce plasma lipid levels. Clinical staging models encourage the use of interventions at each point along the illness pathway to try to reduce the chances of illness progression to later clinical stages such as heart failure or premature death (Scott 2013).

Using a staging approach dictates that any intervention serves two overt goals: one is to manage the current clinical stage; the other, to prevent disease progression (McGorry 2002; Berk 2007; Insel 2009; Hickie 2013; Scott 2013). It is assumed that some individuals can be prevented from progressing to the next stage of illness if interventions are offered. Therefore, there is no absolute threshold for commencing interventions; instead, a sequence of interventions is offered depending on where the individual is located on the spectrum of risk (stage 0 to stage 4). Applying this model to disease management means that interventions are also considered for subthreshold syndromes or presentations that are known to be the antecedents or precursors of severe disorders (Insel 2009). Importantly, the earliest interventions are informed by, but are not the responsibility of, secondary or specialist health services, as many are offered through community or public health initiatives (Scott 2012).

Another important aspect of this model is that treatments are stage-specific, and interventions for the initial stages are not merely a lower dose of the medications prescribed for established disease (Scott 2012). The expectation is that interventions for early stages are usually more benign and will be selected based on having a greater benefitto-risk ratio. Treatments with a greater risk-tobenefit ratio are primarily regarded as the best option for patient with late-stage disorders (Berk 2007). Interestingly, as more individuals at risk of a disorder or in the very earliest stages of illness are detected and treated promptly, the model predicts that the distribution of cases at each illness stage will change, and the proportion of late-stage cases requiring the most complex (and often more expensive) treatment or palliative care is likely to be reduced over time (Scott 2013). This hypothesis is supported by data on the detection, treatment and prognosis of breast cancer, which have changed significantly in recent decades (Lonning 2007).
In summary, clinical staging in medicine represents a substantial improvement over the classic diagnostic approaches used at the beginning of the 20th century. Advances in genetic and biomarker research allow determination of reliable and valid clinicopathological boundaries between stages. Staging improves treatment selection, links the observed clinical phenotype with the extent of disease progression, and guides interventions. The timing of intervention is a key determinant of the success of any specified treatment within a staging model. Clinical staging promotes the notion that interventions should not be delayed until after the individual has crossed a clinical threshold that indicates they are now in a poor prognosis group (i.e. they are highly likely to experience stage 3 or 4 disease). Importantly, staging in medicine has supported the development of novel, stage-appropriate interventions targeting potentially modifiable risk factors to reduce disease progression (Scott 2013).

\section{The origins of staging models for mental disorders}

'Diagnosing schizophrenia or bipolar disorder with the emergence of psychosis [or mania] may be analogous to diagnosing coronary artery disease by myocardial infarction' (Insel 2009).

Historically, the concept of clinical staging was applied to some neuropsychiatric disorders, and models exist for Alzheimer's and Parkinson's diseases. However, it was Fava \& Kellner (1993) who initiated discussions about clinical staging for other severe mental disorders. It should be noted that Fava's views differ from those of other proponents of staging in psychiatry, as the models he describes do not include stage 0 or a latency stage (Cosci 2013). By contrast, several reviews of the implications and benefits of staging (McGorry 2006; Berk 2007; Scott 2013; Kapczinski 2014) all support the notion that, as in general medicine, the inclusion of an asymptomatic, atrisk stage is helpful.

In clinical psychiatry, staging raises awareness of the need to understand disease trajectories and heterotypic continuity; it draws attention to individuals at risk of developing disorders, such as those with a family history and/or subsyndromal presentations; and it actively promotes greater attention to prevention strategies and interventions for individuals at ultra-high risk of developing a severe mental disorder (Conus 2002; McGorry 2002, 2006). It is suggested that international adoption of clinical staging could move general psychiatry away from its traditional focus only on established disease and produce a greater 
appreciation of the need for novel research and treatment strategies that target the early stages of severe mental disorders (Insel 2009; Yung 2010; Hickie 2013; Scott 2013). However, the development of staging paradigms does not have unanimous support (Mahli 2014).

In psychiatry, the identification of at-risk states (or 'stage 0') is hampered by the lack of distinct risk factors and robust endophenotypes or biomarkers (McGorry 2014). There are some studies of neurocognition that may bear fruit, and data from functional magnetic resonance imaging (fMRI) studies indicate that the brain region most commonly found to be abnormal in schizophrenia (the lateral ventricles) is unaffected in stage 1 of psychosis, and less affected in stage 2 than in stage 4 (Pantelis 2009). However, there are still relatively few studies of subthreshold syndromes (Gifford 2016) and currently the use of staging is largely limited to clinical recognition of the early symptomatic presentations of psychosis in individuals seeking help (Yung 2004).

Clinical research has led to the development of criteria for ultra-high-risk (UHR) syndromes (also called at-risk or clinical high-risk) (Yung 2010). For example, three UHR subgroups can be identified on the basis of a range of state and trait risk factors for psychosis, namely, the presence or absence of:

- attenuated psychotic symptoms

- brief limited intermittent psychotic symptoms

- functional deterioration in the context of a family history of psychosis (and probable schizotypal personality).

Several decades of research have shown that about $20-30 \%$ of UHR individuals progress to stage 2 (i.e. develop a psychotic or schizophrenic illness meeting current diagnostic criteria) over about 18 months (Yung 2010; Fusar-Poli 2016). Interestingly, some (but not all) randomised controlled clinical trials and systematic reviews indicate that transition rates can be reduced by almost half by the early introduction of a range of approaches, including cognitive-behavioural therapy (CBT) and novel drug treatments such as omega-3 fatty acids (Liu 2010; Amminger 2015).

\section{Applying clinical staging models to bipolar disorders}

The key issue in broadening the scope of clinical staging from psychotic disorders to the more prevalent affective disorders is that it is potentially more complex to devise a model that considers the varying clinical presentations of the late prodromal stage of bipolar disorder (Scott 2013).
Figure 1 shows one example of a model of staging of bipolar disorder; this model identifies mania as the threshold disorder, so it gives primacy to bipolar disorder type I. However, there are other models, and the one presented here is described as a heuristic model to highlight that it is a proposal that can be used for and refined by future research; it is not meant to be viewed as a definitive model for adult-pattern bipolar disorder. Also, this model does not apply to paediatric or juvenile bipolar disorder, as there is no international consensus on the nature of any relationship between juvenile forms of bipolar disorder and bipolar disorder presenting in adolescents and young adults (Carlson 2011).

As shown in Fig. 1, the latent or preclinical stage of bipolar disorder is best identified by enhanced vulnerability in individuals who have a family history of bipolar disorder. The asymptomatic stage (stage 0) may be followed by the development of non-specific symptoms (stage 1a) and/or may progress further to stage $1 \mathrm{~b}$, which represents subthreshold clinical phenomena. Stage 1b in this model would usually include some symptoms or aspects of current diagnostic concepts (e.g. depression; brief or recurrent hypomania; mood instability; impaired attention; disrupted sleepwake cycle; changes in level of activation or

\begin{tabular}{|c|c|}
\hline Stage 0 & $\begin{array}{l}\text { - Mini-clinical evaluation shows increased risk of bipolar disorder } \\
\text { (recognised by family history, temperamental style, etc.) } \\
\text { - No symptoms currently }\end{array}$ \\
\hline Stage 1a & $\begin{array}{l}\text { - Mild or non-specific symptoms of severe mood or other } \\
\text { disorders } \\
\text { - Mild functional change or decline }\end{array}$ \\
\hline Stage 1b & $\begin{array}{l}\text { - Ultra-high risk } \\
\text { - Moderate but subthreshold symptoms of severe mood disorder } \\
\text { - Functional decline to clinical caseness and/or subtle } \\
\text { neurocognitive changes (e.g in individuals at risk of bipolar } \\
\text { disorder this stage could be a first episode of major depression } \\
\text { with or without evidence of subthreshold mania) }\end{array}$ \\
\hline Stage 2 & $\begin{array}{l}\text { - First episode of mania } \\
\text { - Full-threshold disorder with moderate to severe symptoms and } \\
\text { functional decline (with or without neurocognitive changes) }\end{array}$ \\
\hline Stage 3 & $\begin{array}{l}\text { - Incomplete remission from the first episode } \\
\text { - Recurrence or relapse of bipolar disorder, which stabilises with } \\
\text { treatment at a moderate level of impairment, or with residual } \\
\text { symptoms or neurocognition below the best level achieved } \\
\text { after remission from the first episode }\end{array}$ \\
\hline Stage 4 & $\begin{array}{l}\text { - Multiple relapses accompanied by objective evidence of } \\
\text { worsening in clinical extent and impact of illness } \\
\text { - Severe, persistent or unremitting illness as judged by symptoms } \\
\text { such as treatment-refractory bipolar depression, associated } \\
\text { with neurocognitive deficits and high levels of disability }\end{array}$ \\
\hline
\end{tabular}

Definitions of the stages in a heuristic clinical staging model for bipolar disorders. Adapted from Scott et al (2013). 
energy). Bechdolf et al (2010) and Scott et al (2013) have proposed bipolar disorder at-risk (BAR) criteria that parallel those used to identify UHR individuals in psychosis, namely: subthreshold manic symptoms, cyclothymia with a history of depression, and/or depression with a family history of bipolar disorder. Over 18 months, the transition rate to bipolar disorder in individuals in one of these three at-risk subgroups was $22-25 \%$, compared with a transition rate of less than $1 \%$ in individuals who did not meet any of the at-risk criteria.

Of course, given the relatively recent development of staging models for bipolar disorder, the knowledge base is considerably greater for stage 2 (the first full-threshold stage for the disorder, e.g. mania) onwards. The boundaries between each stage of bipolar disorder are not precisely consistent across models (Berk 2007; Kapczinski 2014; Scott 2013; Duffy 2014), but there is agreement that from stage 2 onwards there is a substantial risk of progression to stable poor-outcome states, characterised by treatment-refractory symptoms, severely impaired functioning and high levels of medical and psychiatric comorbidity. In effect, stages 3 and 4 represent the end stages or final phenotype commonly treated in tertiary referral clinics, and include the subgroup of patients most often included in clinical trials and research studies.

There are potential weaknesses and issues for debate in the heuristic model. The most obvious discussion point in bipolar disorder is where to locate depressive episodes that precede the first manic episode and how to describe subthreshold manic syndromes, especially hypomania. As described in Fig. 1, a depressive episode that preceded the first manic episode or a hypomanic episode could be categorised as stage $1 \mathrm{~b}$. Some readers may worry that this is downgrading the importance of these disorders. Others will dispute whether mild or subtle neurocognitive deficits are detectable in depression in youth. However, it is important to note two things about the location of depressive episodes. First, a depressive episode may indicate an above-average risk of developing a range of mental disorders (and may be a precursor of recurrent unipolar depression, bipolar or psychotic disorder) and not just bipolar disorder. Second, staging models recognise the need to treat stage $1 \mathrm{~b}$, and indeed they advocate the use of appropriate stage-specific interventions to try to prevent progression to stage 2 (threshold diagnosis), so locating depression in stage $1 \mathrm{~b}$ of a bipolar disorder staging model is not indicating that care and treatment should be withheld.
The question of the location of hypomania in the model is also debatable. However, in youth (but probably not in children), there is evidence that hypomania or other subthreshold manifestations of mania are not fixed states, but are strong predictors of future manic episodes (Birmaher 2009; Bechdolf 2010). Research demonstrates that in youth, about one in five individuals with hypomania will progress to mania over about 24 months, so a cross-sectional diagnosis of bipolar disorder type II at this stage does not imply that the same pattern of illness will persist in the future (Ruggero 2010).

The above issues might be resolved if we had a greater understanding of the risk factors, biomarkers or endophenotypes for the onset and progression of bipolar disorder. As in psychosis, most biological research in bipolar disorder targets individuals with established disease. The problem with this approach is that we do not know whether any abnormalities detected are a consequence of the established disease process (e.g. some neurocognitive deficits probably occur post-onset, but there is limited information on any cognitive abnormalities that may increase the risk of onset); whether they are markers of a compensatory change within body systems; or whether a clinical feature is associated with treatment (e.g. hypersomnia may be associated with bipolar depression, or it might arise because of the medication regime prescribed). We have surprisingly little evidence of changes that are causal, and minimal research funding has been directed at identifying biomarkers that predict transition from stage 1b to stage 2 (Scott 2013; McGorry 2014). One of the few prospective studies available, in a small sample of individuals at risk of bipolar disorder (at stage 1b), showed that those who progressed to stage 2 had smaller amygdala volumes than those who did not make the transition to bipolar disorder (Bechdolf 2012). The importance of the amygdala in emotional and mood regulation makes this study worthy of note, but of course larger replication studies are needed before any definitive conclusions can be drawn.

Applying staging models to bipolar disorder may help to refine and improve treatment guidelines. For example, current guidelines, based on crosssectional diagnosis, would probably indicate the prescription of an antidepressant as the most appropriate treatment for a depressive episode in youth. However, a staging model draws attention to the potential trajectory of the presenting condition and encourages clinicians to balance the potential benefits of the standard acute treatment (a traditional antidepressant) against the need to 
prevent disease progression. After consideration of the clinical presentation, this may mean that an antidepressant is prescribed. However, in some cases it might lead the clinician to prescribe a different medication or to offer psychological therapies. For example, these might be viewed as more stage-appropriate first-line treatments if the individual is at high risk of developing bipolar disorder (as the clinician will want to avoid using any treatment that could precipitate hypomanic or manic symptoms).

Research is needed to identify and develop approaches that can be useful for early-stage bipolar disorder. A recent review of psychological therapies for the early stages of bipolar disorder exposed the lack of adequate research, not only on stages 1 and 2, but also a general lack of treatment trials that included younger adults (the average age of trial participants was about 40 years, with an average of about 6 prior bipolar episodes at inclusion). Vallarino et al (2015) also demonstrated that many of the treatments used for adults with established bipolar disorder will need to be adapted quite significantly if we are to improve their efficacy in youth. At present, the most comprehensive research suggests that family-focused treatment can have modest benefits, but findings are inconsistent for CBT and interpersonal therapies. However, it is noteworthy that some of the most established therapies, such as group psychoeducation, have not been studied in young people with emerging mood disorders.

\section{Conclusions}

Clinical staging appears to be a deceptively simple and practical approach in general medicine. However, its utility is obviously greater in disorders where the underlying pathology of the disease is fully understood. In such situations, biomarkers that predict disease onset in at-risk individuals, markers of illness progression and/or biomarkers of treatment response can be differentiated from each other. This level of understanding is not yet available in psychiatry, but clinical staging may help us improve our understanding of the pathophysiological correlates of disease progression and reduce our over-reliance on cross-sectional assessments of symptoms to make diagnoses. Staging models allow us to include other risk factors and known clinical antecedents in the assessment process. Also, it is argued that they offer an informed approach to research and the active promotion of indicated prevention and early intervention strategies (Kapczinski 2014).

A key question for the future is whether the current, disorder-specific staging models might eventually be replaced by a single, transdiagnostic model. In the past decade, researchers have published separate staging models for psychotic, bipolar, depressive, anxiety and eating disorders (McGorry 2006; Berk 2007; Hickie 2013; Scott 2013; Treasure 2015; Verduijn 2015). These have allowed clinicians and researchers to clarify thinking on the phenomenology of each clinical stage and garner evidence for the developmental trajectories of each disorder. However, the early-stage clinical phenotypes of many severe mental disorders are very similar, leading some researchers to argue that the long-term goal should be the development of a single staging model for affective and psychotic disorders (McGorry 2006; Hickie 2013; Scott 2013). Furthermore, there are some transdiagnostic factors that appear to predict transition or disease progression (Grierson 2016). There are many researchers in the field of staging who do not support these proposals (e.g. Duffy 2014), as they feel that important insights will be lost, and that there is insufficient evidence for a pluripotential risk state. Even those who are more supportive of transdiagnostic models accept that these deal poorly with some intervening risk factors, such as substance misuse, which may of course be independent disorders. Lastly, the outcomes of transdiagnostic models need to be clarified, although it is currently suggested that functional level and neuropsychology, rather than any specific clinical symptoms, might be the phenomena used to define stages in a transdiagnostic model (Hickie 2013).

Although there is no consensus on the benefits of a single staging model, the direction of future research means that a transdiagnostic staging model may not be a fanciful idea. For example, the research domain criteria (RDoC) initiative highlights the need to consider psychopathology on a continuum and emphasises that our understanding of liability and comorbidity is more likely to be improved by examining underlying mechanisms that cut across current heterogeneous disorder categories. In keeping with this notion, there is increasing evidence for shared genetic and familial vulnerabilities, common childhood precursors, shared environmental risk factors (e.g. traumatic experiences, substance misuse), concurrent neuropsychological impairment, overlapping structural brain changes and cognitive-emotional dysregulation (Bellivier 2013; Grierson 2016). Taken together, these findings may help us to develop our thinking on how we understand and classify observed clinical phenomena.

From a treatment perspective, staging may help shift the focus towards early intervention 


\section{MCO answers \\ 1 c $\quad 2$ b 3 a $\quad 4$ a $\quad 5$ c}

and a better understanding of the strengths and weaknesses of using more generic interventions in young adults with undifferentiated symptoms of a possible severe mental disorder. If less specific treatments were equally effective in preventing transitions to bipolar disorder or transitions to psychotic disorders, this would suggest that, in the early stages, the commonalities between disorders at the same stage (e.g. stage $1 \mathrm{~b}$ of psychosis and stage $1 \mathrm{~b}$ of bipolar disorder) may exceed differences that exist within the same disorder at different stages (e.g. stage 1b compared with stage 3 of bipolar disorder). Alternatively, if such generic approaches to early clinical presentations fail to prevent transition to full-syndrome disease, it may be argued that the underlying pathophysiology is different for each disorder even at very early stages, indicating the need for more specific treatments even for at-risk individuals.

\section{References}

Amminger GP, Schäfer MR, Schlögelhofer M, et al (2015) Longer-term outcome in the prevention of psychotic disorders by the Vienna omega-3 study. Nature Communications, 11: 7934.

Bechdolf A, Nelson B, Cotton SM, et al (2010) A preliminary evaluation of the validity of at-risk criteria for bipolar disorders in help-seeking adolescents and young adults. Journal of Affective Disorders, 127: $316-20$

Bechdolf A, Wood SJ, Nelson B, et al (2012) Amygdala and insula volumes prior to illness onset in bipolar disorder: a magnetic resonance imaging study. Psychiatry Research, 201: 34-9.

Bellivier F, Geoffrey P, Scott J, et al (2013) Biomarkers of bipolar disorder: specific or shared with schizophrenia? Frontiers in Bioscience, 5: 845-63.

Berk M, Conus P, Lucas N, et al (2007) Setting the stage: from prodrome to treatment resistance in bipolar disorder. Bipolar Disorder, 9: 671-8.

Birmaher B, Axelson D, Goldstein B, et al (2009) Four-year longitudinal course of children and adolescents with bipolar spectrum disorders: the Course and Outcome of Bipolar Youth (COBY) study. American Journal of Psychiatry, 166: 795-804.

Carlson GA (2011) Will the child with mania please stand up? British Journal of Psychiatry, 198: 171-2.

Conus P, McGorry PD (2002) First-episode mania: a neglected priority for early intervention. Australian \& New Zealand Journal of Psychiatry, 36: $158-72$.

Cosci F, Fava GA (2013) Staging of mental disorders: systematic review. Psychotherapy and Psychosomatics, 82: 20-34.

Duffy A (2010) The early natural history of bipolar disorder: what have we learned from longitudinal high-risk research. Canadian Journal of Psychiatry, 55: 477-85.

Duffy A (2014) Toward a comprehensive clinical staging model for bipolar disorder: integrating the evidence. Canadian Journal of Psychiatry, 59: $659-66$.

Fava G, Kellner R (1993) Staging: a neglected dimension in psychiatric classification. Acta Psychiatrica Scandinavica, 87: 225-30.

Fusar-Poli P, Cappucciati M, Borgwardt S, et al (2016) Heterogeneity of psychosis risk within individuals at clinical high risk: a meta-analytical stratification. JAMA Psychiatry, 73: 113-20.

Gifford G, Crossley N, Fusar-Poli P, et al (2016) Using neuroimaging to help predict the onset of psychosis. Neurolmage, 145: 209-17.
Gonella J, Hornbrook M, Louis D (1984) Staging of disease: a case-mix measurement. JAMA, 251: 637-44.

Grierson A, Naismith S, Hickie I (2016) The role of rumination in illness trajectories in youth: linking trans-diagnostic processes with clinical staging models. Psychological Medicine, 46: 2467-84.

Hickie IB, Scott EM, Hermens DF, et al (2013) Applying clinical staging to young people who present for mental health care. Early Intervention in Psychiatry, 7: 31-43.

Insel T (2009) Translating scientific opportunity into public health impact: a strategic plan for research on mental illness. Archives of General Psychiatry, 66: 128-33

Kapczinski F, Magalhães PV, Balanzá-Martinez V, et al (2014) Staging systems in bipolar disorder: an International Society for Bipolar Disorders Task Force Report. Acta Psychiatria Scandinavica, 130: 354-63.

Kim-Cohen J, Caspi A, Moffitt T (2003) Prior juvenile diagnoses in adults with mental disorder: developmental follow-back of a prospectivelongitudinal cohort. Archives of General Psychiatry, 60: 709-19.

Liu P, Parker AG, Hetrick SE, et al (2010) An evidence map of interventions across premorbid, ultra-high risk and first episode phases of psychosis. Schizophrenia Research, 123: 37-44.

Loftus J, Etain B, Scott J (2016) What can we learn from offspring studies in bipolar disorder? BJPsych Advances, 22: 176-85

Lonning PE (2007) Breast cancer prognostication and prediction: are we making progress? Annals of Oncology, 18 (suppl 8): 3-7.

Malhi GS, Rosenberg DR, Gershon S (2014) Staging a protest! Bipolar Disorders, 16: 776-9.

McGorry PD (2002) The recognition and optimal management of early psychosis: an evidence-based reform. World Psychiatry, 1: 76-83.

McGorry PD, Hickie IB, Yung AR, et al (2006) Clinical staging of psychiatric disorders: a heuristic framework for choosing earlier, safer and more effective interventions. Australian \& New Zealand Journal of Psychiatry. 40: 616-22.

McGorry P, Keshavan M, Goldstone S, et al (2014) Biomarkers and clinical staging in psychiatry. World Psychiatry, 13: 211-23.

Pantelis C, Yucel M, Bora E, et al (2009) Neurobiological markers of illness onset in psychosis and schizophrenia: the search for a moving target. Neuropsychology Reviews, 19: 385-98.

Ruggero CJ, Carlson GA, Kotov R, et al (2010) Ten-year diagnostic consistency of bipolar disorder in a first-admission sample. Bipolar Disorders, 12: 21-31.

Scott J, Hickie I, McGorry P (2012) Pre-emptive psychiatry: pipe dream or realistic outcome of clinical staging models. Neuropsychiatry, 2: 263-6.

Scott J, Leboyer M, Hickie I, et al (2013) Clinical staging in psychiatry: a cross-cutting model of diagnosis with heuristic and practical value. British Journal of Psychiatry, 202: 243-5.

Treasure J, Stein D, Maguire S (2015) Has the time come for a staging model to map the course of eating disorders from high risk to severe enduring illness? An examination of the evidence. Early Intervention in Psychiatry, 9: 173-84.

Vallarino M, Henry C, Etain B, et al (2015) An evidence map of psychosocial interventions for the earliest stages of bipolar disorder. Lancet Psychiatry, 2: 548-63.

Verduijn J, Milaneschi Y, van Hemert AM, et al (2015) Clinical staging of major depressive disorder: an empirical exploration. Journal of Clinical Psychiatry, 76: 1200-8.

Wright JR Jr (2012) Albert C. Broders' paradigm shifts involving the prognostication and definition of cancer. Archives of Pathology \& Laboratory Medicine, 136: 1437-46.

Yung AR, Phillips LJ, Yuen HP, McGorry PD (2004) Risk factors for psychosis in an ultra high-risk group: psychopathology and clinical features. Schizophrenia Research, 67: 131-42.

Yung AR, Nelson B, Thompson A, et al (2010) The psychosis threshold in Ultra High Risk (prodromal) research: is it valid? Schizophrenia Research 120: 1-6. 
MCQs

Select the single best option for each question stem

1 There is no published staging model available for:

a ischaemic heart disease

b breast cancer

c attention-deficit hyperactivity disorder

d psychosis

e rheumatoid arthritis.

\section{Current diagnostic criteria:}

a have a high level of validity

b have a high level of reliability

c are most reliable and valid when applied to individuals in the peak age range for the onset of severe mental disorders (about 15-25 years of age)

$d$ were developed primarily from clinical assessments of individuals presenting in community and primary care settings e can be used to describe illness trajectories.
3 When using staging models:

a the treatments selected for early-stage disease are usually high benefit and low risk

b the same medications are used across clinical stages, but the dose and duration of treatment differ according to stage

c treatment is not advised for individuals below stage 2

d the model cannot predict the course or outcome of a disorder

e the boundaries between each stage are best defined by the duration of illness.

4 In staging models for bipolar disorders:

a stage 0 is best represented by individuals with a family history of bipolar disorder(s)

b stage 1 can include a range of syndromes such as paediatric or juvenile bipolar disorder

c stage 2 includes subsyndromal presentations such as minor depression

d stage 3 represents the traditional diagnostic threshold e stage 4 indicates a manic episode with psychotic features.

5 Randomised controlled trials of interventions for the early stages of mental disorders demonstrate that:

a in bipolar disorders, low-dose lithium can be used for stage $1 \mathrm{~b}$

b in bipolar disorders, group psychoeducation is the most widely researched intervention for stages 1 and 2

c in psychosis, omega-3 fatty acids might help to prevent transition from stage $1 \mathrm{~b}$ (an ultra-highrisk state) to stage 2

$\mathrm{d}$ in bipolar disorders, cognitive-behavioural therapy is the preferred intervention for individuals at stage $1 \mathrm{~b}$

e in psychosis, family-focused treatment is the psychological treatment of choice. 